\title{
Análisis espacial y del entorno físico de accidentes de tránsito en la ciudad de Resistencia, Chaco, Argentina
}

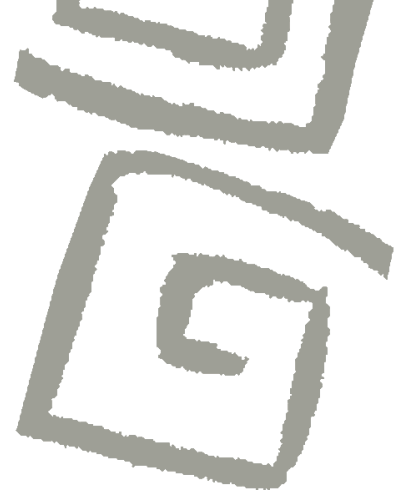

\author{
Geospatial and environmental analysis of road traffic \\ accidents in the city of Resistencia, Argentina
}

Martín Chaparro ${ }^{1}$, Akram Hernández-Vásquez², Alejandro Parras ${ }^{3}$

${ }^{1}$ Médico, Especialista en Epidemiología. Estudiante de Posgrado, Facultad de Medicina, Universidad de Buenos Aires, Buenos Aires, Argentina. $\square$ (iD)

${ }^{2}$ Médico Cirujano, Magíster en Gestión y Políticas Públicas. Profesor, Facultad de Ciencias de la Salud, Universidad Privada del Norte, Lima, Perú. $₫$ (iD

${ }^{3}$ Licenciado en Geografía, Magíster en Teledetección y Sistemas de Información Geográfica. Profesor, Facultad de Humanidades, Universidad Nacional del Nordeste, Chaco, Argentina. $\Delta$ iD
RESUMEN Los accidentes de tránsito constituyen un problema emergente en ciudades con alta movilidad y baja planificación urbana. No existe suficiente evidencia en Argentina acerca de la relación entre el entorno y la ocurrencia de accidentes. Por ello, realizamos un análisis espacial de ocurrencia y estimamos el riesgo de ocurrencia de accidentes en el año 2012 y su posible asociación con las características del entorno físico en la ciudad de Resistencia, provincia del Chaco, Argentina. Para el análisis espacial de los accidentes se utilizó la estimación de densidad kernel. Luego, a través de un estudio observacional y analítico se procedió a analizar los factores asociados a la ocurrencia de los accidentes. Los resultados muestran que existen tres zonas críticas (noroeste, centro y sur) con mayor frecuencia de accidentes. Los factores del entorno que estuvieron asociados con la ocurrencia de accidentes fueron la presencia de iluminación vial (23\% menor), árbol próximo a la calzada (47\% mayor), la presencia de semáforo (28\% mayor), avenidas (122\% mayor) y disposición curva de la calzada (129\% mayor). Este estudio ubica a la ciudad de Resistencia en una situación de vulnerabilidad urbana, no solo por el contexto socioeconómico donde se encuentra sino por el desigual desarrollo territorial que presenta respecto a las ciudades vecinas, que propicia un entorno desfavorable.

PALABRAS CLAVES Sistemas de Información Geográfica; Análisis Espacial; Accidentes de Tránsito; Ambiente; Argentina.

\begin{abstract}
Traffic accidents are an emerging problem in cities with high mobility and little urban planning. Evidence is lacking in Argentina regarding the relationship between the environment and accident occurrence; we therefore conducted a geospatial analysis and estimated the risk of accidents and their possible association with the characteristics of the physical environment in the city of Resistencia, Argentina occurring in 2012. Kernel density estimates were used for the spatial distribution of accidents and in parallel an observational, analytical study was carried out to analyze the factors associated with accident occurrence. The results show three critical areas (in the northwest, center and south of the city) with greater accident frequency. Environmental factors that were associated with the occurrence of accidents were the presence of street lighting $(23 \%$ greater), the presence of a tree close to the road ( $47 \%$ greater), the presence of a traffic light ( $28 \%$ greater), and if the road was a major avenue (122\% greater) and had curves $(129 \%$ greater). This study shows the city of Resistencia to be in a situation of urban vulnerability due not only to its socioeconomic status but also to the unequal development compared to neighboring cities, a reality that fosters an unfavorable environment.
\end{abstract}

KEY WORDS Geographic Information Systems; Spatial Analysis; Traffic Accidents; Environment; Argentina. 


\section{INTRODUCCIÓN}

Las muertes por lesiones no intencionales se han constituido en un importante problema de salud pública por el continuo incremento de la mortalidad a lo largo del tiempo ${ }^{(1)}$. Solo en el año 2010, el 9,6\% del total de muertes en el mundo fueron atribuidas a lesiones no intencionales, de las cuales 1,3 millones fueron por accidentes de tránsito ${ }^{(2)}$. Se estima que, a nivel mundial, el costo generado por los accidentes de tránsito asciende a más de 500.000 millones de dólares, de los cuales 65.000 millones corresponderían a países de bajos y medianos ingresos, en donde ocurren más del $90 \%$ de las muertes ${ }^{(3,4)}$.

En Argentina, durante 2007 se registraron 5.914 muertes por accidentes de tránsito ${ }^{(5)}$. Respecto de los años de vida perdidos por muerte prematura, los accidentes de tránsito pasaron de ser la sexta causa en 2005 a ubicarse en la cuarta posición en $2015^{(6)}$. Algunas provincias del país como Santa Fe, Chaco y Córdoba reportan elevadas cifras de accidentes que generan un incremento en la morbimortalidad total ${ }^{(7)}$. Debido a que el escenario descrito podría persistir en el tiempo, es necesario estudiar más a fondo los factores locales asociados a la ocurrencia de accidentes.

En el sistema del tránsito, el medio físico constituye uno de los elementos fundamentales a la hora de determinar los factores de riesgos que entran en juego durante la movilidad cotidiana de la población. Diversos estudios revelan cómo influyen las condiciones materiales de la vía pública en el comportamiento de los sujetos y en la generación de accidentes de tránsito ${ }^{(8,9)}$. Estas condiciones se relacionan con la densidad del alumbrado público vial, el diseño de las vías, la presencia de cruces peatonales, entre otras ${ }^{(10,11,12)}$. Sin embargo, en muchas ciudades argentinas todavía no se ha estudiado si las condiciones del entorno influyen de manera positiva o negativa en la ocurrencia de accidentes de tránsito.

En la actualidad, diversos sistemas de información geográfica (SIG) aplicados a las investigaciones en salud con perspectiva espacial o territorial se utilizan para el estudio de los accidentes de tránsito. En Argentina, algunos estudios han utilizado estos sistemas y demuestran su utilidad para explorar patrones de accidentes o para identificar zonas de mayor riesgo ${ }^{(13,14,15)}$. Por ello, los SIG podrían ser de utilidad en áreas con elevada frecuencia de accidentes de tránsito con la finalidad de implementar posibles medidas de prevención.

El objetivo general de este trabajo es identificar la presencia de patrones de ocurrencia de accidentes de tránsito mediante un análisis geoespacial de los puntos de ocurrencia de accidentes durante el año 2012, y analizar el riesgo de ocurrencia de los accidentes en su posible asociación con las características del entorno físico en la ciudad de Resistencia, provincia de Chaco, Argentina.

\section{MATERIALES Y MÉTODOS}

\section{Diseño y ámbito del estudio}

Se realizó un estudio transversal, espacial y analítico a partir de registros policiales de accidentes de tránsito ocurridos durante el año 2012, tomando como área de estudio la ciudad de Resistencia, capital de la provincia del Chaco, en el noreste de Argentina. Resistencia, la ciudad más poblada de la provincia y su principal centro económico y cultural, es también uno de los centros más importantes de la región. Se ubica en el departamento de San Fernando y cuenta con una población de 390.874 habitantes ${ }^{(16)}$.

\section{Variable dependiente o de resultado}

Se consideró como variable de resultado, para el análisis tanto espacial como del ambiente físico, la frecuencia de accidentes. Se consideraron dos unidades de estudio: como unidad espacial de análisis se tomó la dirección postal donde ocurrió el accidente y, como unidad de observación, el entorno para el análisis de los factores ambientales, definido como el espacio que se extiende a 
20 metros a la redonda del punto de accidente. Este valor fue elegido tomando como base la distancia mínima de frenado de un vehículo según la regla del cuadrado (considerando una velocidad promedio de 40 $\mathrm{km} / \mathrm{h})^{(17)}$. Se consideró como accidente cualquier contacto no intencionado entre dos o más unidades móviles dentro de una vía de circulación sin tener en consideración el tipo y/o desenlace final (lesiones/muerte).

\section{Variables independientes}

Las variables independientes consideradas para el análisis del entorno fueron: a) tipo de calzada: que comprende las avenidas, calles y rutas según la tipificación vial del plano catastral y que fueron verificadas mediante observación directa; b) origen de la calzada: se consideró como punto de partida la plaza central (principal) o una calzada principal (secundaria), según el plano catastral, que fue verificado mediante observación directa; c) presencia de iluminación vial: cualquier dispositivo de alumbrado artificial (postes o faroles) que se encontraba dentro del entorno, con verificación de su funcionamiento por un informante clave; d) presencia de ciclovía: cualquier carril de una vía pública que ha sido señalizado apropiadamente para este propósito o una vía independiente en la que se permite el tránsito de bicicletas, próximo al entorno donde ocurrió el evento; e) árbol próximo a la calzada: se consideró como próximo a aquellos que se encontraban a una distancia menor o igual a $20 \mathrm{~m}$ del área del accidente con dificultad para la visualización de los alrededores; f) disposición de la calzada: según la forma que adoptaba la calzada se tipificó en recta, curva y sinuosa; g) calzada pavimentada: si la calzada presentaba asfalto según inspección visual del entorno del evento; h) reductores de velocidad sobreelevados: cualquier dispositivo colocado sobre la superficie de la calzada a lo largo de ciertos tramos de la vía, en el entorno del accidente (por ejemplo, paso peatonal sobreelevado, lomo de burro, etc.); i) presencia de semáforo: en la intersección más próxima al entorno del accidente.

\section{Técnicas e instrumentos de recolección de datos}

Las variables fueron recolectadas en una planilla de registro ad hoc de las observaciones en el periodo de julio a diciembre de 2012. Sobre la base de los registros de la policía local, se inspeccionaron los entornos en donde se habían registrado accidentes de tránsito y se geocodificaron las direcciones postales a través de dos etapas: en una primera se procedió a la codificación a través del programa Batchgeo y Google Maps y, en una segunda etapa, en aquellos sitios que no fue posible identificar un punto por estos métodos, se procedió in situ a registrar las coordenadas a través de GPS marca Garmin, modelo eTrex Legend ${ }^{\circledR} \mathrm{HCx}$.

Se elaboraron mapas a través de Google Maps, tomando diferentes fracciones censales de la ciudad e identificando los sitios (puntos) donde ocurrieron los accidentes. Se le entregó un mapa a cada encuestador delimitando las fracciones censales de interés junto con un formulario ad hoc para la recolección de los datos del entorno. Se elaboró una base de datos en el programa Microsoft Excel ${ }^{\circledR} 2013$.

En esta última etapa, se entrenó al personal en el manejo de GPS y llenado del registro de observaciones mediante un instructivo para cada operador. Se llevó a cabo una prueba piloto, tomando cinco puntos seleccionados al azar, para realizar la inspección visual de su entorno. Luego del proceso de recolección, los datos fueron analizados en dos fases.

\section{Análisis de datos}

En la primera fase, se usó el programa ArcGIS Desktop versión 10.4 para proyectar cada punto georreferenciado en formato shapefile (.shp) y se utilizó el método de estimación de densidad kernel gaussiana por $\mathrm{km}^{2}$, con ancho de banda predeterminado y tamaño 
de celda de 30, para estimar la relación de los puntos, clasificar en intervalos iguales y localizar aquellas áreas con alta (áreas rojas), mediana (áreas amarillas) y baja densidad (áreas azules) de eventos. Esta técnica analiza la relación entre varios puntos o eventos geográficos (por ejemplo, puntos georreferenciados de accidentes), de manera que los puntos más cercanos generan áreas de mayor frecuencia (densidad) dentro de un área determinada. Se generó una capa de densidad con los 1.342 puntos. Los archivos creados en formato shapefiles fueron vinculados y proyectados a mapas de base de OpenStreetMap.

Seguidamente, en la segunda fase, se buscaron asociaciones con las variables ambientales mediante el paquete estadístico Stata $^{\oplus}$ v14.1. Las variables categóricas fueron descritas mediante frecuencias absolutas y relativas. Para el análisis estadístico bivariado y multivariado se usaron modelos lineales generalizados (GLM) de Poisson y función de enlace log tomando como variable de resultado la frecuencia de accidentes de tránsito. Teniendo en cuenta el carácter multifactorial de causalidad de los accidentes de tránsito y que el análisis se corresponde con un corte transversal, nuestro modelo de regresión no fue construido con el fin de analizar su capacidad predictiva, sino para analizar las relaciones y asociaciones entre la variable dependiente y las independientes. Para el análisis multivariado, se incluyeron aquellas variables que mostraron asociación mínima con la variable dependiente y se calculó la razón de frecuencia media esperada de accidentes (RFM) con intervalos de confianza del $95 \%$. Se consideró, para todos los análisis, un valor $p<0,05$ como estadísticamente significativo.

\section{Consideraciones éticas}

El presente estudio no requirió la aprobación de un comité de ética por tratarse de un análisis de datos secundarios (registros policiales) que no permite identificar a los participantes y/o involucrados en los accidentes de tránsito.

\section{RESULTADOS}

De los 2.500 eventos registrados en 2012, se alcanzaron a georreferenciar 1.342 accidentes de tránsito ocurridos en la planta urbana de la ciudad de Resistencia. De ellos, se procedió al relevamiento ambiental de 462 puntos, ya que muchos accidentes corresponden al mismo entorno (Figura 1).

La estimación de la densidad kernel permitió identificar patrones de distribución espacial que orientan la interpretación de los resultados. Para ello, la densidad de la ocurrencia de dichos accidentes quedó representada en cuatro clases: a) muy alta densidad, b) alta densidad, c) mediana densidad, y d) baja densidad. De esta manera, en la Figura 2 se evidencia que las densidades muy altas se ubican en el sector sureste de la ciudad de Resistencia. En dicho sector, se configuran dos áreas: una representada por la intersección de la Av. 9 de Julio y la calle Ildefonso Pérez y la otra representada por la intersección de la Av. Juan José Castelli y la Av. Arribalzaga. Respecto de las densidades altas de accidentes de tránsito, se identificaron tres lugares representados en el sector noroeste, tales como la Av. 25 de Mayo (altura 2500), Av. Carlos María de Alvear (altura 3300, límite administrativo entre las ciudades de Fontana y Resistencia), y el sector sureste representado por la intersección de la Av. Edison y la Av. Arribalzaga. Asimismo, se identificaron seis áreas con densidades medias de accidentes de tránsito (Figura 2).

En el análisis del entorno de los patrones identificados con muy alta densidad de accidentes se observaron las siguientes características:

1)Av. 9 de Julio y calle Pérez: su disposición responde a la configuración ortogonal de la trama urbana. No hay semáforos ni reductores de velocidad, no hay señalización horizontal (para el cruce peatonal). La calle Pérez (con calzada de asfalto), si bien tiene asignada una sola dirección correspondiente a la salida del Barrio Luzuriaga de la ciudad de Resistencia, culturalmente es 
utilizada en doble sentido. Respecto de la iluminación vial, hay presencia de postes de luz con adecuado funcionamiento.

2) Av. Juan José Castelli y Av. Arribalzaga (o calle 21): es una intersección constituida por dos avenidas principales de la ciudad, con presencia de semáforos, que están ubicados antes del cruce de la intersección y la senda peatonal. El área de influencia de este sector representa una de las zonas más pobladas de la ciudad.
Los entornos de las densidades altas de accidentes de tránsito presentaron las siguientes características:

1)Av. 25 de Mayo, altura 2500: representa la prolongación de la avenida (asfaltada) y el enlace con Ruta Nacional $N^{0} 11$, no se visualizaron semáforos ni señalización horizontal ni vertical. Esta es una avenida de doble sentido (ingreso y salida a la ciudad de Resistencia), respecto del eje de ingreso

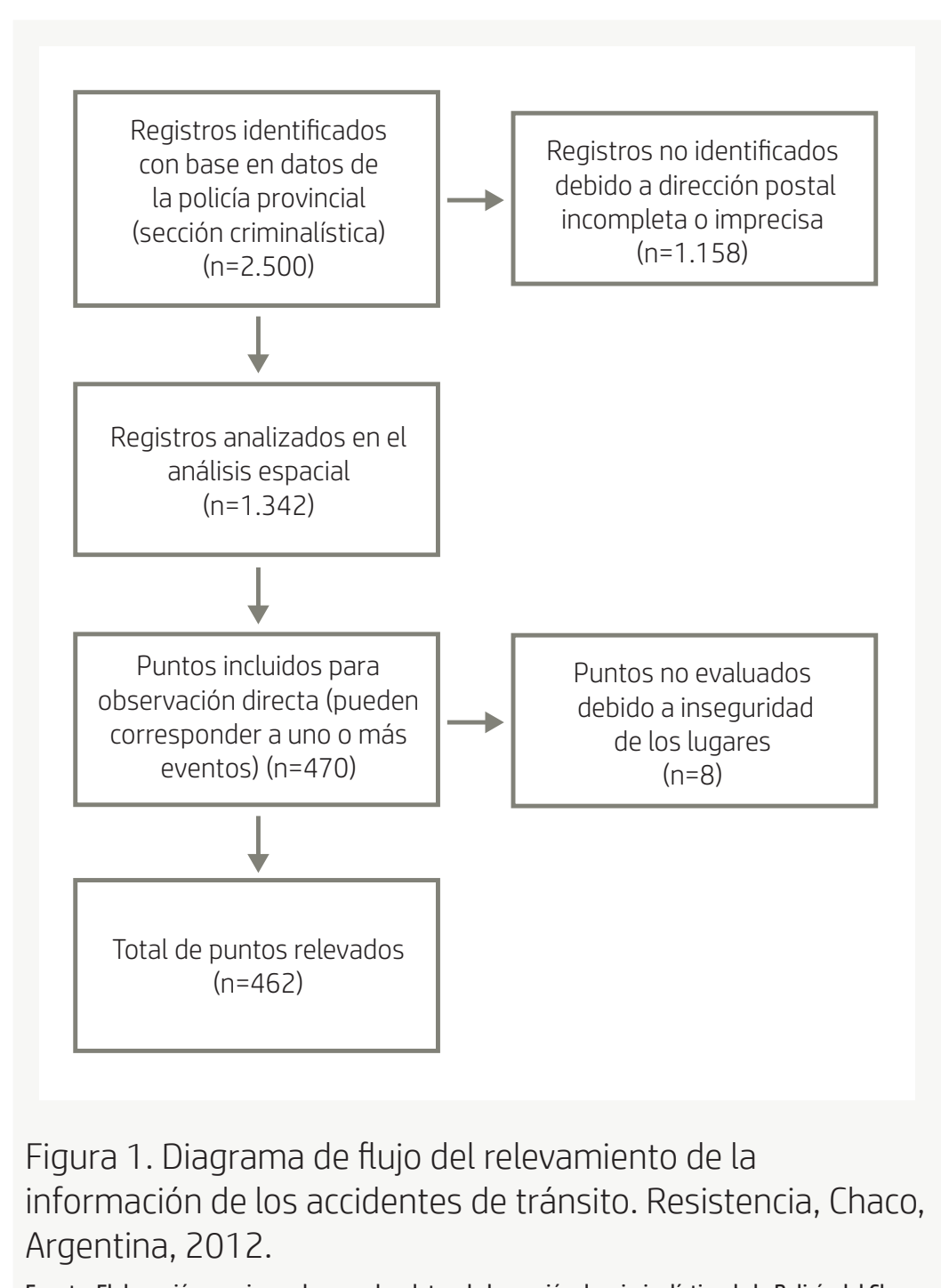

Salud Colectiva | Universidad Nacional de Lanús | ISSN 1669-2381 | EISSN 1851-8265 | doi: 10.18294/sc.2018.1207 


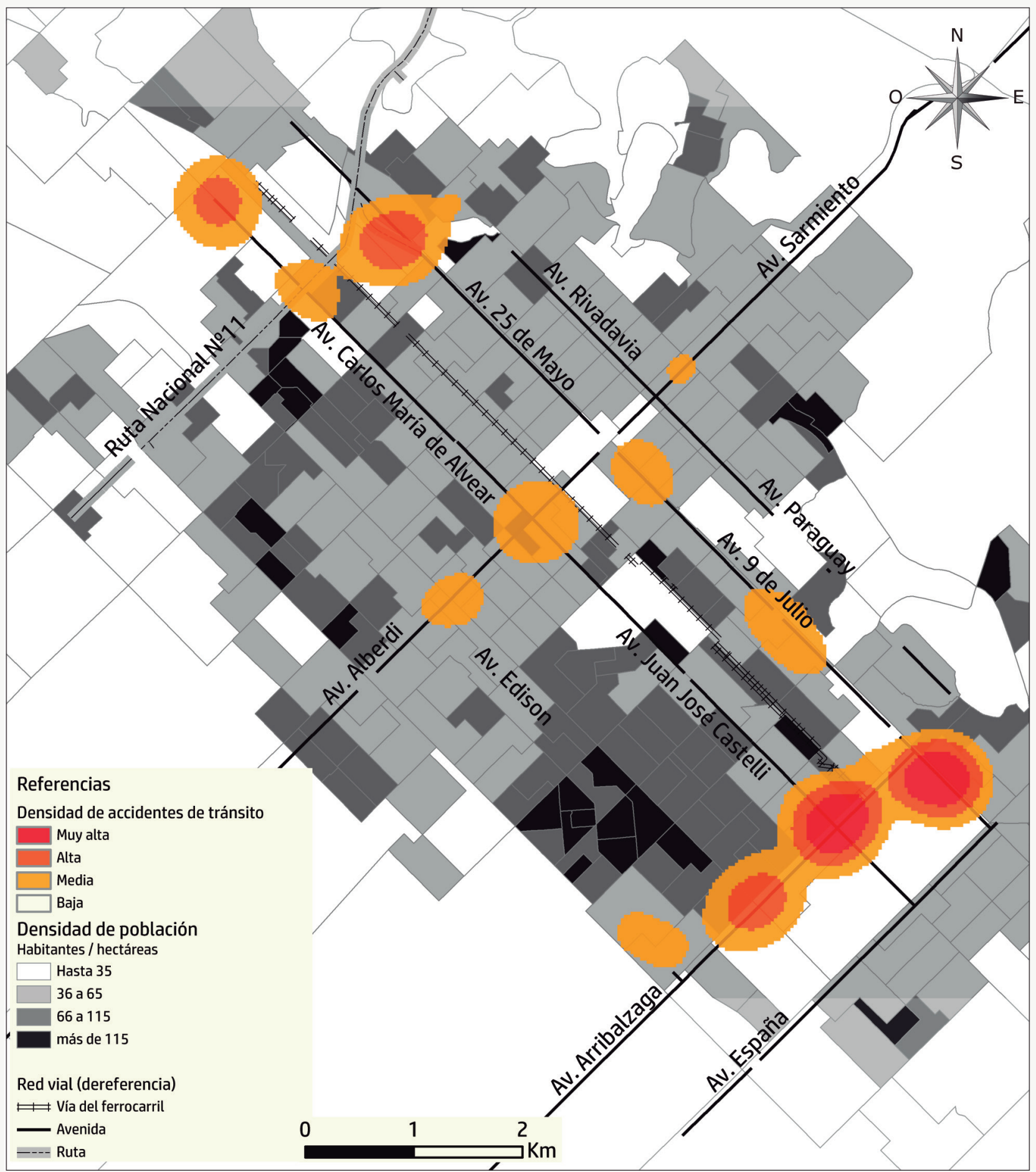

Figura 2. Análisis espacial de la densidad de accidentes de tránsito en la planta urbana de la ciudad de Resistencia, Chaco, Argentina ( $N=1.342)$.

Fuente: Elaboración propia con base en los datos de la sección de criminalística de la Policía del Chaco y Censo Nacional de Población, Hogares y Viviendas 2010. 
Tabla 1. Características del entorno de los accidentes según las variables del ambiente en Resistencia, Chaco, Argentina, 2012. ( $\mathrm{N}=462)$.

\begin{tabular}{|c|c|c|}
\hline Características & Frecuencia & $\%$ \\
\hline \multicolumn{3}{|l|}{ Facilitadores y barreras } \\
\hline Presencia de lluminación vial* & 330 & 71,74 \\
\hline Presencia de ciclovía & 46 & 9,96 \\
\hline Árbol próximo a la calzada & 63 & 13,64 \\
\hline \multicolumn{3}{|l|}{ Restrictores de velocidad } \\
\hline Presencia de semáforo & 47 & 10,17 \\
\hline Reductores de velocidad sobreelevados** & 63 & 13,73 \\
\hline \multicolumn{3}{|l|}{ Calzada } \\
\hline Calzada pavimentada & 338 & 73,16 \\
\hline \multicolumn{3}{|l|}{ Tipo de calzada } \\
\hline Avenida & 290 & 62,77 \\
\hline Calle & 168 & 36,36 \\
\hline Ruta & 4 & 0,87 \\
\hline \multicolumn{3}{|l|}{ Origen de la calzada } \\
\hline Principal & 168 & 36,36 \\
\hline Secundaria & 294 & 63,64 \\
\hline \multicolumn{3}{|l|}{ Disposición de la calzada } \\
\hline Recta & 433 & 93,72 \\
\hline Curva & 17 & 3,68 \\
\hline Sinuosa & 12 & 2,60 \\
\hline
\end{tabular}

a la ciudad, que está conectada con la calle Campias (asfaltada). No se observaron señales de tránsito que den cuenta de esta conexión ni postes de alumbrado público.

2) Av. Carlos María de Alvear, altura 3300: es la intersección de esta avenida (asfaltada) con la calle Lago Nahuel Huapi (de tierra) que constituye la línea sobre la cual se apoya el límite administrativo entre las ciudades de Resistencia y Fontana. En el entorno no se evidenció presencia de semáforos, ni señalización horizontal, ni vertical. No existen elementos que obstaculicen la visión durante la circulación, tales como árboles, carteles, etc.
3) Av. Edison y Av. Arribalzaga: ambas avenidas son de tierra, no cuentan con semáforos ni presentan ningún tipo de señalización horizontal o vertical. No se visualizaron elementos identificatorios de alumbrado público.

En la Tabla 1, respecto de las características generales del entorno, se observa que, de los lugares con ocurrencia de accidentes, un $71,74 \%$ presentó iluminación vial; un $73,16 \%$, calzada pavimentada; el 62,77\% eran avenidas, el $63,64 \%$ de tipo secundarias y el $93,72 \%$ de disposición recta. Respecto de los atenuantes de velocidad, el 10,17\% tenía 
semáforo próximo y, el $13,73 \%$, reductores sobreelevados. El 13,64\% de los entornos presentó un árbol próximo a la calzada.

Respecto de los factores asociados (Tabla 2), en el análisis bivariado, la presencia de ciclovía, reductores sobreelevados, calzada pavimentada y el origen de la calzada no se asoció de manera estadísticamente significativa con la frecuencia de accidentes de tránsito.
Sin embargo, tanto en el análisis bivariado como en el multivariado se encontró que la presencia de iluminación vial, árbol próximo a la calzada, la presencia de semáforo, tipo y disposición de la calzada se asoció de manera estadísticamente significativa.

La presencia de iluminación vial disminuyó la frecuencia media de accidentes en un $23 \%$; sin embargo, la presencia de

Tabla 2. Asociación entre las características del entorno y la frecuencia de accidentes en cada lugar de ocurrencia, Resistencia, Chaco, Argentina, 2012. ( $N=460)$.

\begin{tabular}{|c|c|c|c|c|c|c|}
\hline \multirow{2}{*}{ Característica } & \multicolumn{3}{|c|}{ RFM crudo } & \multicolumn{3}{|c|}{ RFM ajustado } \\
\hline & RFM & IC $95 \%$ & Valor de $p$ & RFM & IC $95 \%$ & Valor de $p$ \\
\hline \multicolumn{7}{|c|}{ Presencia de iluminación vial } \\
\hline Sí & 0,78 & $0,68-0,89$ & $<0,001$ & 0,77 & $0,67-0,88$ & $<0,001$ \\
\hline $\mathrm{No}^{*}$ & - & - & - & - & - & - \\
\hline \multicolumn{7}{|c|}{ Presencia de ciclovía } \\
\hline Sí & 0,94 & $0,75-1,16$ & 0,570 & - & - & - \\
\hline $\mathrm{No}^{*}$ & - & - & - & - & & \\
\hline \multicolumn{7}{|c|}{ Árbol próximo a la calzada } \\
\hline Sí & 1,46 & $1,24-1,71$ & $<0,001$ & 1,47 & $1,24-1,73$ & $<0,001$ \\
\hline $\mathrm{No}^{*}$ & - & - & - & - & - & - \\
\hline \multicolumn{7}{|c|}{ Presencia de semáforo } \\
\hline Sí & 1,26 & $1,05-1,53$ & 0,013 & 1,28 & $1,05-1,56$ & 0,012 \\
\hline $\mathrm{No}^{*}$ & - & - & - & - & - & - \\
\hline \multicolumn{7}{|c|}{ Reductores de velocidad sobreelevados } \\
\hline Sí & 0,96 & $0,80-1,16$ & 0,720 & - & - & - \\
\hline $\mathrm{No}^{*}$ & - & - & - & - & - & - \\
\hline \multicolumn{7}{|c|}{ Calzada pavimentada } \\
\hline Sí & 0,94 & $0,82-1,10$ & 0,460 & - & - & - \\
\hline $\mathrm{No}^{*}$ & - & - & - & - & - & - \\
\hline \multicolumn{7}{|l|}{ Tipo de calzada } \\
\hline Avenida & 3,74 & $2,52-5,54$ & $<0,001$ & 2,22 & $1,42-3,45$ & $<0,001$ \\
\hline Calle & 1,25 & $1,09-1,43$ & 0,001 & 1,23 & $1,07-1,42$ & 0,003 \\
\hline Ruta* & - & - & - & - & - & - \\
\hline \multicolumn{7}{|c|}{ Origen de calzada } \\
\hline Secundaria & 0,96 & $0,85-1,10$ & 0,630 & - & - & - \\
\hline Principal* & - & - & - & - & - & - \\
\hline \multicolumn{7}{|c|}{ Disposición de calzada } \\
\hline Recta & 0,82 & $0,52-1,28$ & 0,390 & 0,71 & $0,45-1,12$ & 0,148 \\
\hline Curva & 2,64 & $2,13-3,28$ & $<0,001$ & 2,29 & $1,80-2,92$ & $<0,001$ \\
\hline Sinuosa* & - & - & - & - & - & - \\
\hline
\end{tabular}

Fuente: Elaboración propia con base en datos de la observación directa.

*Valor de referencia. RFM= Razón de frecuencia media. IC 95\%= Intevalo de confianza del 95\% 
árbol próximo aumentó un 47\% la frecuencia media de accidentes, y la presencia de semáforos, un $28 \%$. Además, en las avenidas, la frecuencia media de accidentes fue 2,2 veces mayor comparada con los accidentes que ocurrieron en las rutas y la frecuencia media en las calles fue 1,23 veces mayor. En las calzadas con disposición curva, la frecuencia media de accidentes también fue mayor comparada con la de calzadas sinuosas $(2,29$ veces mayor).

\section{DISCUSIÓN}

Los hallazgos de este estudio muestran áreas vulnerables de frecuencia de accidentes en tres sectores claves de la ciudad (noroeste, centro y sur) y este aumento está relacionado con algunos factores del entorno que influyen de manera significativa.

Desde el punto de vista geográfico, Resistencia ocupa un lugar central y estratégico respecto de otras ciudades contiguas del noreste argentino tales como Fontana, Barranqueras y Puerto Vilelas. Todas estas, junto a la primera, integran lo que se conoce como el Área Metropolitana del Gran Resistencia. Esta situación geográfica representa una dimensión de análisis que merece ser considerada en los estudios de movilidad cotidiana, por su relación directa con las características estructurales de la circulación que evidencian el desequilibrio territorial en estos términos ${ }^{(18)}$.

El Área Metropolitana del Gran Resistencia es un fuerte centro administrativo, comercial y de prestación de servicios, que concentra la mayor oferta de servicios, así como los mejores indicadores de vivienda, educación y de salud en una provincia con elevados índices de pobreza. Esta centralidad y las mejores condiciones de vida, se reproducen y profundizan en la relación desigual que Resistencia ejerce con los otros municipios que integran esta área ${ }^{(19)}$.

En estos términos, la Av. 9 de Julio y la Av. Juan José Castelli (paralelas entre sí, de acuerdo a la configuración de la trama urbana) junto con la Av. Soberanía Nacional, la Av. 25 de Mayo y la Av. Carlos María de Alvear representan los ejes de comunicación principales para vincular la ciudad de Resistencia con las ciudades vecinas. Esta situación explica la configuración de las altas densidades de ocurrencia de accidentes de tránsito en los sectores señalados previamente. Dicha justificación está basada en la idea de que sobre estas arterias circulan diversos modos de transporte, tales como vehículos no motorizados (como bicicletas) y los vehículos a motor (motos, autos, ómnibus) acompañados del mayor flujo de personas que se trasladan. Otros estudios Ilevados a cabo en Argentina demostraron que la región del centro y norte del país son las zonas más afectadas por accidentes, hecho que se vincula en parte al crecimiento demográfico y el aumento del parque automotor ${ }^{(13)}$.

Nuestros resultados de asociación entre la presencia de iluminación vial y la frecuencia repetida de accidentes se asemeja a lo hallado en una revisión sistemática, en la que tres ensayos compararon la iluminación de las calles con un área control en cuanto al total de accidentes; el riesgo relativo (RR) agrupado fue de 0,45 [IC95\% $(0,29-0,69)]^{(11)}$. Asimismo, en otra revisión, los autores reportaron que en dos estudios tipo antes-después para reducir los atropellamientos en las noches, al incrementarse la iluminación sobre zonas de cruce urbano se encontró un descenso de la frecuencia de eventos del $57 \%$, en Israel, y del 59\%, en Australia ${ }^{(20)}$.

Un aspecto a tener en cuenta para la comparación es ajustar según el momento del día en que ocurrieron los eventos (mañana, tarde o noche) ya que es determinante al momento de atribuir el riesgo a este factor. Este podría ser un potencial confundidor de la asociación considerando que el mayor número de accidentes ocurre durante la noche o a primeras horas de la mañana, cuando el efecto de la iluminación cobra importancia ${ }^{(10,21)}$. Por ello, es indispensable conocer los puntos críticos de accidentes de tránsito y relevar su estado actual de iluminación, ya que al existir una correlación entre bajas condiciones de iluminación y frecuencia de accidentes se pueden 
implementar mejoras en dichos puntos como medida preventiva ${ }^{(22)}$.

Respecto de la presencia de árboles próximos a la calzada, en nuestro estudio se encontró que los lugares con ausencia de árboles presentaron menor número de accidentes. De forma similar, en un estudio de casos y controles que evaluó la relación entre distintos factores ambientales y la ocurrencia de lesiones en ciclistas, hallaron un odds ratio ajustado de 3,25 para los sitios con más de un árbol por cuadra ${ }^{(23)}$.

Según el tipo de calzada, se observó que el $62,77 \%$ de los lugares donde ocurrieron accidentes se trataba de avenidas con un incremento en 2,22 veces la frecuencia de eventos comparado con las rutas. Este resultado está alineado con diversos estudios que mostraron que este tipo de vías presentan mayor frecuencia de accidentes de tránsito, tal vez, debido a las velocidades que se imponen en este tipo de calzadas, lo cual podría favorecer la ocurrencia de accidentes ${ }^{(12,24)}$. Además, se debe tener en cuenta que existen factores combinados que podrían potenciar su efecto respecto de la frecuencia de accidentes, tal es el caso de los lugares con circulación por vías de alta velocidad sumado a su bajo nivel de iluminación ${ }^{(22)}$. Otro factor relacionado con las calzadas, es su disposición curva que, en nuestro análisis, fue el mayor factor para la frecuencia de accidentes. Este hallazgo ha sido reportado en algunos estudios, incluso la dirección derecha o izquierda de la curva puede condicionar la ocurrencia de accidentes ${ }^{(25,26,27)}$.

Por su parte, en nuestro estudio, la presencia de un semáforo cercano al entorno del evento incrementó la frecuencia de accidentes de tránsito. Si bien este tipo de elemento de señalización tiene como función controlar y regular el tránsito, pensamos que un alto porcentaje de accidentes ocurrieron por la falta de cumplimiento de sus indicaciones o señales. Algunos estudios reportaron tasas de incumplimiento de las señales del semáforo por parte de peatones de hasta un $35 \%$ que incrementarían el riesgo de sufrir un accidente ${ }^{(28)}$. En un estudio multicéntrico, en el que uno de los sitios fue Resistencia, los autores destacan el alto incumplimiento de las señales de los semáforos y que las nomas del tránsito son transgredidas en general ${ }^{(29)}$. Bajo este escenario, consideramos necesario que se profundice la investigación de este tipo de hallazgo para determinar las posibles causas que podrían estar generando una mayor ocurrencia de accidentes ante la presencia de semáforos.

Diversos estudios han demostrado que la implementación de dispositivos de detección de excesos de velocidad (cámaras de detección, radares o láser) reducen entre un $40 \%$ y un $45 \%$ la probabilidad de accidentes fatales y de lesiones causadas por el tránsito ${ }^{(30,31,32)}$. Por lo tanto, las intervenciones encaminadas a reducir la velocidad del tránsito resultan esenciales y efectivas para prevenir lesiones y las muertes. Sin embargo, todos estos estudios se llevaron a cabo en países de altos ingresos. En Perú, por ejemplo, se pueden encontrar varias intervenciones de este tipo, aunque no siempre implementadas en los sitios necesarios o de manera integral, a fin de alcanzar el máximo efecto ${ }^{(33)}$.

Es fundamental la adopción de estrategias integrales, un plan integrado de gestión de manejo responsable, que incluya actividades educativas, señalización masiva, y la aplicación de dispositivos de bajo costo para la detección de incumplimiento de las normas de tránsito. Yannis et al. mencionaron que un plan integrado de gestión de la velocidad, para ser eficaz y exitoso, no solo depende del seguimiento sistemático de la ejecución del plan y de la seguridad vial sino que también necesita un fuerte apoyo político de los gobiernos locales. Las intervenciones dirigidas a la reducción de lesiones por tránsito deberían integrarse en tres pilares fundamentales: intervenciones educativas, legales y de ingeniería o infraestructura ${ }^{(32,34)}$.

Una potencial limitación fue que el relevamiento se realizó un año posterior a la ocurrencia de los eventos, con la posibilidad de que las condiciones del ambiente hayan variado. Es poco probable que esto haya ocurrido debido a las características permanentes de los factores evaluados, dado que no se anunciaron nuevas obras de infraestructura. 
Otro aspecto a considerar es la mala calidad del registro policial de localización de los accidentes, con el potencial riesgo de haber presentado un patrón de concentración espacial diferente u otro tipo de relación estadística con las variables independientes incluidas. Sin embargo, consideramos esta limitación como un indicador importante que brinda la oportunidad de incorporar sistemas de información en tiempo real que permitan corroborar nuestros hallazgos. Por otro lado, se debe mencionar que la técnica utilizada para el análisis espacial (densidad kernel) se basa en la mayor frecuencia de ocurrencia de eventos sin establecer pruebas de significancia estadística que permitan establecer con certeza si los patrones encontrados se distribuyen de manera aleatoria. Asimismo, una limitación a tener en cuenta respecto a la metodología de análisis de los factores fue la falta de información con relación a los niveles de circulación según los tipos de calzadas que permita realizar ajustes de los conteos de accidentes de tránsito en el modelo de regresión de Poisson.

Considerando las mencionadas limitaciones, este estudio aporta información valiosa para la planificación urbana ya que no existe suficiente evidencia en países de bajos y medianos ingresos respecto de la relación del entorno y la ocurrencia de accidentes. Conocer las áreas calientes de ocurrencia permitirá la vigilancia y monitoreo por parte del gobierno local y orientar intervenciones efectivas para la reducción de las lesiones en estos sitios.

\section{CONCLUSIONES}

Este estudio ubica a la ciudad de Resistencia en una situación de vulnerabilidad urbana, no solo por su contexto socioeconómico, sino por el desigual desarrollo territorial que presenta respecto de las ciudades vecinas, que propicia un entorno desfavorable. La planificación urbana es deficiente debido a la falta de iluminación vial y de señalización, y por la presencia de obstáculos como árboles próximos a la calzada podrían ser factores facilitadores que predisponen a la mayor frecuencia de accidentes. Además, la presencia de semáforos en los puntos de mayor número de accidentes alerta sobre la baja adherencia a las normas de control vial por parte de los conductores.

\section{AGRADECIMIENTOS}

Este estudio fue financiado a través del Programa de Becas de Investigación "Carrillo-Oñativia" 2013, otorgada por el Ministerio de Salud de la Nación.

\section{REFERENCIAS BIBLIOGRÁFICAS}

1. Peden M. Global collaboration on road traffic injury prevention. International Journal of Injury Control and Safety Promotion. 2005;12(2):85-91.
2. Lozano R, Naghavi M, Foreman K, Lim S, Shibuya K, Aboyans V, et al. Global and regional mortality from 235 causes of death for 20 age groups in 1990 and 2010: a systematic analysis for the Global Burden of Disease Study 2010. The Lancet. 2012;380(9859):2095-2128.

3. Organización Mundial de la Salud. Informe mundial sobre prevención de los traumatismos causados por el tránsito [Internet]. Ginebra: OMS; 2004 [citado 20 jul 2016]. Disponible en: https:// tinyurl.com/y9ujotkk. 
4. GBD 2013 Mortality and Causes of Death CoIlaborators. Global, regional, and national age-sex specific all-cause and cause-specific mortality for 240 causes of death, 1990-2013: a systematic analysis for the Global Burden of Disease Study 2013. The Lancet. 2015;385(9963):117-171.

5. Ubeda C, Espitia-Hardeman V, Bhalla K, Borse NN, Abraham JP, Dellinger A, et al. National burden of road traffic injuries in Argentina. International Journal of Injury Control and Safety Promotion. 2012;19(1):9-18.

6. Institute for Health Metrics and Evaluation. Country profile: Argentina [Internet]. Seattle: Institute for Health Metrics and Evaluation; [citado 10 oct 2016]. Disponible en: http://www.healthdata. org/argentina.

7. Ministerio de Transporte Presidencia de la Nación. Observatorio Vial: Informes Estadísticos [Internet]. Buenos Aires: Ministerio de Transporte Presidencia de la Nación ; [citado 10 oct 2016]. Disponible en: https://tinyurl.com/yc72m4jl.

8. Fuentes CM, Hernandez V. Spatial environmental risk factors for pedestrian injury collisions in Ciudad Juarez, Mexico (2008-2009): implications for urban planning. International Journal of Injury Control and Safety Promotion. 2013;20(2):169-178.

9. Lankarani KB, Heydari ST, Aghabeigi MR, Moafian G, Hoseinzadeh A, Vossoughi M. The impact of environmental factors on traffic accidents in Iran. Journal of Injury and Violence Research. 2014;6(2):64-71.

10. Plainis S, Murray IJ, Pallikaris IG. Road traffic casualties: understanding the night-time death toll. Injury Prevention. 2006;12(2):125-128.

11. Beyer FR, Ker K. Street lighting for preventing road traffic injuries. The Cochrane Database of Systematic Reviews. 2009;(1):Cd004728.

12. Peden M, Scurfield R, Sleet D, Mohan D, Hyder AA, Jarawan E, Mathers C, editors. World report on road traffic injury prevention [Internet]. Switzerland: World Health Organization; 2004 [citado 10 oct 2016]. Disponible en: https:// tinyurl.com/ycmm494e.

13. Leveau CM. Spatial variations in motorcycle registrations and the mortality of motorcycle users due to traffic injuries in Argentina. Salud Colectiva. 2013;9(3):353-362.

14. Leveau CM, Ubeda C. Deaths from road injuries in Argentina: a spatial analysis for the 2001-
2009 period. Revista Panamericana de Salud Pública. 2012;31(5):439-442.

15. Macías GR, Almeida Filho N, Alazraqui $M$. Análisis de las muertes por accidentes de tránsito en el municipio de Lanús, Argentina: 1998-2004. Salud Colectiva. 2010;6(3):313-328.

16. Instituto Nacional de Estadística y Censos. Censo Nacional de Población, Hogares y Viviendas del año 2010 [Internet]. Buenos Aires: INDEC; 2010 [citado 20 jul 2016]. Disponible en: https://tinyurl.com/y9gvm522.

17. Dirección General de Tráfico, Ministerio del Interior de España. La velocidad [Internet]. España: Dirección General de Tráfico, Ministerio del Interior de España; 2017 [citado 5 may 2017]. Disponible en: https://tinyurl.com/y76k3m3q.

18. Valdés P. La ordenación de espacios metropolitanos: consideraciones sobre el eje Gran Resistencia-Gran Corrientes. Cuaderno Urbano: Espacio, Cultura y Sociedad. 2009;8(8):181-204.

19. Alcalá L, Scornik M. Movilidad y accesibilidad en el Gran Resistencia: principales problemas y desafíos. Revista Transporte y Territorio. 2015;13:8-35.

20. Rodriguez-Hernandez JM, Campuzano-Rincón JC. Primary prevention measures for controlling pedestrian injuries and deaths and improving road safety. Revista de Salud Pública. 2010;12(3):497509.

21. Sullivan JM, Flannagan MJ. The role of ambient light level in fatal crashes: inferences from daylight saving time transitions. Accident Analysis and Prevention. 2002;34(4):487-498.

22. Jägerbrand $A K$, Sjöbergh J. Effects of weather conditions, light conditions, and road lighting on vehicle speed. SpringerPlus. 2016;5:505.

23. Romanow NTR, Couperthwaite AB, McCormack GR, Nettel-Aguirre A, Rowe $\mathrm{BH}$, Hagel BE. Environmental determinants of bicycling injuries in Alberta, Canada. Journal of Environmental and Public Health. 2012;2012: 487681.

24. Hijar M, Carrillo C, Flores M, Anaya R, Lopez $\mathrm{V}$. Risk factors in highway traffic accidents: a case control study. Accident Analysis and Prevention. 2000;32(5):703-709.

25. Othman S, Thomson R, Lannér G. Identifying critical road geometry parameters affecting crash rate and crash type. Annals of Advances in Automotive Medicine. 2009;53:155-165. 
26. Othman S, Thomson R, Lannér G. Are driving and overtaking on right curves more dangerous than on left curves? Annals of Advances in Automotive Medicine. 2010;54:253-264.

27. Elvik R. Does the influence of risk factors on accident occurrence change over time? Accident Analysis and Prevention. 2016;91:91-102.

28. King MJ, Soole D, Ghafourian A. Illegal pedestrian crossing at signalised intersections: incidence and relative risk. Accident Analysis and Prevention. 2009;41(3):485-490.

29. Geldstein R, Bertoncello R, Peterlini C, Thomas C. Aspectos demográficos y sociales de los accidentes de tránsito en áreas seleccionadas de la Argentina; diagnóstico y aportes para el diseño de políticas y programas de prevención [Internet]. Buenos Aires: CONAPRIS, Ministerio de Salud y Ambiente; 2006 [citado 18 oct 2016]. Disponible en: https://tinyurl.com/y939oof3.

30. Wilson C, Willis C, Hendrikz JK, Bellamy N. Speed enforcement detection devices for preventing road traffic injuries. The Cochrane Database of Systematic Reviews. 2006;(2):Cd004607.

31. Wilson C, Willis C, Hendrikz JK, Le Brocque R, Bellamy N. Speed cameras for the prevention of road traffic injuries and deaths. The Cochrane Database of Systematic Reviews. 2010;(11):Cd004607.

32. Olsen JR, Mitchell R, Mackay DF, Humphreys DK, Ogilvie D. Effects of new urban motorway infrastructure on road traffic accidents in the local area: a retrospective longitudinal study in Scotland. Journal of Epidemiology and Community health. 2016;70(11):1088-1095.

33. Quistberg DA, Miranda JJ, Ebel B. Reducing pedestrian deaths and injuries due to road traffic injuries in Peru: interventions that can work. Revista Peruana de Medicina Experimental y Salud Pública. 2010;27(2):248-254.

34. Sadeghi-Bazargani H, Saadati M. Speed management strategies: a systematic review. Bulletin of Emergency and Trauma. 2016;4(3):126-133.

FORMA DE CITAR

Chaparro M, Hernández-Vásquez A, Parras A. Análisis espacial y del entorno físico de accidentes de tránsito en la ciudad de Resistencia, Chaco, Argentina. Salud Colectiva. 2018;14(1):139-151. doi: 10.18294/sc.2018.1207

Recibido: 31 de octubre de 2016 | Versión final: 8 de mayo de 2017 | Aprobado: 7 de julio de 2017

Este obra está bajo una licencia de Creative Commons Reconocimiento-NoComercial 4.0 Internacional. Reconocimiento - Permite copiar, distribuir y comunicar públicamente la obra. A cambio, se debe reconocer y citar al autor original. No Comercial - Esta obra no puede ser utilizada con finalidades comerciales, a menos que se obtenga el permiso. 